\title{
RESTABELECIMENTO DE SISTEMAS DE DISTRIBUIÇÃO UTILIZANDO FLUXO DE POTÊNCIA ÓTIMO
}

\author{
Thiago T. Borges* \\ thiago_tb@hotmail.com \\ Sandoval Carneiro Jr* \\ sandoval@dee.ufrj.br
}

\author{
Paulo A. N. Garcia* \\ paulo.garcia@ufjf.edu.br
}

José L. R. Pereira ${ }^{\dagger}$

jluiz@ieee.org

*Universidade Federal do Rio de Janeiro - RJ

†Universidade Federal de Juiz de Fora - MG

\begin{abstract}
Distribution System Restoration Using Optimal Power Flow

This paper proposes a methodology to solve the Distribution System restoration problem after a fault. This is a Mix Integer Non-Linear optimization problem which poses considerable challenges to traditional methods. The approach described in this paper models the discrete variables of the problem as continuous functions, thus the problem can be solved using the Primal-Dual Interior Point method. The proposed methodology uses graph theory to identify isolated areas and loops in the system. System switches are closed based on a index, obtained from an Optimum Power Flow (OPF) algorithm. The problem is solved step-by-step, providing a feasible switching operation sequence. The proposed method are tested and comparisons show the efficcience and robustness of the algorithm.
\end{abstract}

KEYWORDS: Distribution Restoration, Reconfiguration, Step by Step approach, Interior Points, Optimum Power Flow.

Artigo submetido em 14/10/2011 (Id.: 1386)

Revisado em 12/12/2011, 29/02/2012

Aceito sob recomendação do Editor Associado Prof. Antonio Carlos Zambroni de Souza

\section{RESUMO}

Este artigo apresenta uma metodologia para solução do problema de restabelecimento de Sistemas de Distribuição de Energia após a ocorrência de um defeito. Trata-se de um problema de programação não linear inteira mista de difícil solução pelos métodos tradicionais. Na metodologia proposta, as variáveis discretas do problema são modeladas como uma função contínua, permitindo que o problema possa ser resolvido utilizando-se o Método Primal-Dual de Pontos Interiores. No processo de solução, inicialmente utiliza-se teoria de grafos para determinar as áreas isoladas e os laços no sistema. Em seguida, as chaves são fechadas com base em um índice obtido através do Fluxo de Potência Ótimo (FPO). O problema é resolvido passo a passo de tal forma que uma sequência viável de operação é fornecida no final do processo. Testes e comparações são realizados mostrando a eficiência da metodologia proposta.

PALAVRAS-CHAVE: Restabelecimento, Defeitos, Reconfiguração, Algoritmo Passo a Passo, Pontos Interiores.

\section{INTRODUÇÃO}

As interrupções no fornecimento dos Sistemas de Distribuição de Energia Elétrica (SDE) são na sua maioria imprevisíveis e inevitáveis. Esses problemas ocorrem após um defeito no sistema devido a diversos fatores. De acordo com Gomes (2008), esses problemas ocorrem devido a diversos fatores entre eles: 
- Ações da natureza: descargas atmosféricas, vendavais, tornados, terremotos, geadas;

- Ações do homem: queimadas, vandalismo, erros de projeto, erros de operação.

Após a ocorrência de um defeito, os equipamentos de proteção detectam o problema e isolam uma parte do sistema. Em sistemas com alimentadores radiais (que é o caso mais comum) esta operação resulta no desligamento de alguns ramos do alimentador a jusante da área afetada. Nessas circunstâncias deve-se inicialmente isolar o segmento defeituoso do sistema, abrindo as chaves ao redor dessa área.

O próximo passo nesse processo é restabelecer o suprimento de energia nos ramos não defeituosos através da reconfiguração dos alimentadores. O processo de reconfiguração envolve o uso de chaves normalmente abertas (NA) e normalmente fechadas (NF) entre as áreas isoladas e os alimentadores adjacentes. Os operadores do SDE devem primeiramente isolar o defeito, detectar a área desconectada do sistema e em seguida selecionar as chaves que devem ser fechadas e abertas (Fukuyama and Chiang, 1995).

Conforme Adibi et al. (1987), o problema de restauração do sistema elétrico depois de um colapso total ou parcial, é praticamente tão velho como a indústria de energia elétrica em si. Em Bryson and Hayward (1940), foram discutidos os problemas e procedimentos para o restabelecimento do sistema metropolitano da Duquesne Light Company, Pittsburgh, Estados Unidos. Muitas companhias de eletricidade têm desenvolvido, ao longo dos anos, regimes de restauração do sistema que satisfazem suas necessidades particulares de operação.

De acordo com Hsu et al. (1992) um plano de restabelecimento efetivo dos SDE deve possuir as seguintes características:

- ser executado no menor tempo possível;

- restabelecer a maior quantidade de carga possível;

- mínimo número de operações de chaveamento;

- os limites de tensão e corrente não devem ser violados;

- a estrutura radial deve ser mantida.

Por vários anos, os SDE foram planejados, construídos e têm sido operados na configuração radial. A baixa confiabilidade desses sistemas é melhorada adicionando chaves de interconexão (chaves tie) entre os alimentadores. Recentemente, com a abertura do mercado de energia, os governos têm tomado ações para reduzir o número e a duração das interrupções nos SDE. Logo, as concessionárias de energia elétrica estão abandonando o tradicional sistema radial e adotando uma estrutura malhada mais flexível e confiável (Celli et al., 2004). Em um futuro próximo, a radialidade do sistema de distribuição pode deixar de ser uma restrição para os planos de restabelecimento. Assim, as metodologias para resolver este problema devem estar preparadas para lidar com essa questão.

De acordo com Delbem et al. (2000), outros objetivos também podem ser considerados, de acordo com as exigências de cada companhia. Por exemplo, pode-se exigir que seja mantida a carga equilibrada entre alimentadores, reduzindo o nível de queda de tensão e minimizando perdas ou que se evite planos que precisem de operação de chaves distantes umas das outras no sistema de distribuição.

Normalmente, o problema de restabelecimento é muito complicado e demorado para ser resolvido. Isso ocorre devido ao fato dos sistemas urbanos de distribuição serem de grande escala e possuírem um grande número de chaves candidatas para serem operadas (Aoki et al., 1989). Este aspecto pode ser entendido facilmente com uma análise matemática simples. Configurações de sistemas de distribuição normalmente são representadas pelo conjunto dos estados das chaves. Essas configurações podem ser descritas por um vetor conforme Equação (1).

$$
x=\left[x_{1}, \ldots, x_{n}\right]
$$

Onde:

$x_{n} \quad$ Representa o estado na n-ésima chave do sistema (Aberta $\left(x_{n}=0\right)$ ou fechada $\left(x_{n}=1\right)$;

$n$ Representa o número de chaves manobráveis do sistema.

Desta forma, $2^{n}$ configurações de rede podem ser geradas até que uma possível configuração viável seja encontrada (que forneça energia para a área desenergizada). Isso pode requerer um tempo de processamento muito elevado, principalmente se o sistema de distribuição em consideração for de grande dimensão. Portanto, métodos que analisem todos os possíveis vetores não são práticos, até mesmo para sistemas de distribuição pequenos (Delbem et al., 2000).

Durante a operação real dos SDEs somente um número reduzido de alternativas é analisado devido ao tempo limitado para o planejamento e à dificuldade de se analisar todas as alternativas possíveis de maneira ótima, considerando-se os objetivos do problema (Asakura et al., 2000). Para o restabelecimento, estes tempos são ainda mais reduzidos.

No contexto atual dos sistemas de distribuição, processos de restauração do suprimento de energia baseados exclusi- 
vamente na experiência de operadores humanos não são inteiramente confiáveis, além de serem de implementação difícil. Portanto, para atender os objetivos e critérios cada vez mais rigorosos, estratégias de reconfiguração para o restabelecimento mais eficiente são necessárias. Para reduzir a área sem energia de forma mais eficiente e auxiliar na tomada de decisão dos operadores, uma decisão assistida por computador deve ser utilizada (Kumar, Gupta and Gupta, 2006).

Tendo em vista a importância do restabelecimento das redes de distribuição de energia elétrica para a confiabilidade do sistema e satisfação dos consumidores, existem na literatura diversos trabalhos publicados sobre métodos e algoritmos para solução deste problema. Em Sarma et al. (1994) é proposto um método de Busca Exaustiva que avalia todas as topologias factíveis que satisfaçam as restrições do problema. No entanto, devido à dimensão elevada de sistemas reais e de grande porte, uma solução prática requer o emprego de métodos de busca heurísticos, baseados em um conjunto de regras estabelecidas de acordo com as características do problema que permitem reduzir o espaço de busca. Entre esses métodos, pode-se citar Busca Heurística (Hsu et al., 1992; Lin and Chin, 1998; MathiasNeto et al., 2010; Miu et al., 1998; Morelato and Monticelli, 1989; Shirmohammadi, 1992), Branch-Exchange (Lei et al., 2000; Peponis and Papadopoulos, 1995) e Sistemas Especialistas (Chen et al., 2002; Liu et al., 1988; Tsai and $\mathrm{Wu}, 2002)$. Algoritmos baseados em meta-heurísticas como Busca Tabu (Duque and Morinigo, 2006) também tem sido usados na solução desse problema.

Da mesma forma, algorítmos baseados em sistemas inteligentes, como Algorítmos Genéticos (Bretas et al., 2001; Chavali et al., 2002; Fukuyama and Chiang, 1995; Kumar, Das and Sharma, 2006; Luan et al., 2002; Mun et al., 2001; Xianchao et al., 2010), Lógica Fuzzy (Chen, 2010; Delbem et al., 2000; Hsu and Kuo, 1994; Huang, 2003; Kaewmanee and Sirisumrannukul, 2011; Kuo and Hsu, 1993; Lim et al., 2006; Popovic and Popovic, 2004; Rodriguez and Vargas, 2005; Trovato et al., 1999), Sistemas Imunológicos (Li et al., 2009), Enxame de Partículas (Tian et al., 2010; YiXiong et al., 2011) e Redes Neurais Artificiais (Hsu and Huang, 1995) podem ser usados para resolução do problema.

Métodos de otimização discreta também têm sido usados. $\mathrm{O}$ método do gradiente dual efetivo é apresentado Aoki et al. (1989). Em Momoh and Caven (2003) é proposta uma metodologia de otimização que combina a técnica de Branch and Bound para programação inteira com o Método de Pontos Interiores.

Nessa linha de pesquisa, o presente trabalho apresenta um algoritmo para restabelecimento dos SDE utilizando-se Fluxo de Potência Ótimo (FPO). As chaves do sistema são mode- ladas como funções contínuas (Gomes et al., 2006; Oliveira et al., 2009) e incorporadas no problema de FPO. O fechamento das chaves NA para restabelecer o sistema é baseado em um índice fundamentado na posição da chave e nos resultados obtidos do FPO. A cada passo da solução do problema uma chave é fechada, provendo uma sequência viável de operação. Quando um laço é formado, o algoritmo indica uma chave NF para ser aberta. Para solução do problema de FPO utiliza-se o Método Primal Dual de Pontos Interiores (MPI) (Granville et al., 1996; Karmarkar and Ramakrishnan, 1991). $\mathrm{O}$ algoritmo proposto neste trabalho foi testado em sistemas de distribuição encontrados na literatura e os resultados são comparados para avaliar o seu desempenho e validade.

\section{METODOLOGIA PROPOSTA}

O restabelecimento de Sistemas de Distribuição de Energia Elétrica (SDE) pode ter múltiplos objetivos. Entre eles podese citar a restauração da maior quantidade de carga, minimização do custo da energia não distribuída e minimização do corte de carga. Além disso, todos esses objetivos devem estar aliados ao atendimento dos limites técnicos de tensão e fluxo nas linhas. No presente artigo foi utilizada como função objetivo a restauração da maior quantidade de carga no sistema.

Dessa forma, o problema de FPO proposto para restabelecimento ótimo do sistema de distribuição pode ser formulado como:

$$
\operatorname{Max} \mathrm{FOB}=\left(\sum_{k \in \Omega r}\left(\gamma_{k} \cdot P l_{k}\right)\right)
$$

s.a.

$$
\begin{aligned}
P g_{k}-P l_{k}+\sum_{m \in \Omega k} C H_{k m}\left(x_{k m}\right) \cdot P_{k m} & =0 \quad\left(\lambda p_{k}\right) \\
C H_{k m}\left(x_{k m}\right) \cdot Q_{k m} & =0 \quad\left(\lambda q_{k}\right) \\
\bar{Z}^{\min } \leq \bar{Z} & \leq \bar{Z}^{\max }
\end{aligned}
$$

Onde:

FOB Representa a função objetivo;

$\Omega r$ Representa o conjunto de barras na área desenergizada;

$\gamma_{k}$ Representa o corte de carga associado às barras da área desenergizada do sistema;

$\Omega_{k}$ Representa o conjunto de barras conectadas à barra $k$;

$P g_{k}$ Representa a geração de potência ativa na barra $k$;

$P l_{k}$ Representa a carga ativa na barra $k$;

$\lambda p_{k}$ Representa o multiplicador de Lagrange associado à equação de balanço de potência ativa (3a); 
$Q g_{k}$ Representa a geração de potência reativa na barra $k$;

$Q l_{k}$ Representa a carga reativa na barra $k$;

$Q_{k m}$ Representa o fluxo de potência reativa no circuito $k-$ $m$;

$\lambda q_{k}$ Representa o multiplicador de Lagrange associado à equação de balanço de potência reativa (3b);

$\bar{Z}$ Representa o vetor das demais variáveis do FPO;

$\bar{Z}^{\text {min }}, \bar{Z}^{\text {max }}$ Representam os limites mínimo e máximo das variáveis $\bar{Z}$, respectivamente.

A função $C H_{k m}\left(x_{k m}\right)$ representa a modelagem da chave entre as barras $k$ e $m$ no FPO. Estas chaves podem assumir somente dois valores: 0 (chave aberta) ou 1 (chave fechada). Portanto, uma chave pode ser representada pela função degrau unitário (Gomes et al., 2006). No entanto, a função degrau apresenta uma descontinuidade no ponto de mudança de estado, no qual, esta função não é diferenciável e, portanto, não pode ser modelada no FPO convencional. Como alternativa, no trabalho é utilizada a função sigmóide similar a apresentada em da Silva et al. (2008) e Oliveira et al. (2010), dada pela Equação (4) e ilustrada na Figura 1.

$$
C H_{k m}\left(x_{k m}\right)=\frac{e^{\alpha \cdot x_{k m}}-1}{e^{\alpha \cdot x_{k m}}+1} \quad x_{k m} \geq 0
$$

Onde:

$$
\begin{array}{ll}
x_{k m} & \begin{array}{l}
\text { Representa a variável de otimização } \\
\text { associada à chave manobrável acoplada } \\
\text { ao circuito } k-m ;
\end{array} \\
C H_{k m}\left(x_{k m}\right) & \begin{array}{l}
\text { Representa o valor de posição da chave } \\
\text { manobrável do circuito } k-m .
\end{array}
\end{array}
$$

Para a função sigmoide utilizada, foi adotado o parâmetro $\alpha=1$ conforme descrito em da Silva et al. (2008). A Figura 1 mostra que o argumento da função sigmóide $\left(x_{k m}\right)$ varia essencialmente na faixa de zero a 20.

A Equação (2 define a função objetivo do FPO, e está associada à maximização da carga ativa nas barras inicialmente desenergizadas do sistema. A variável $\gamma_{k}$ varia entre 0 e 1 , representando o corte de carga nas barras não alimentadas do sistema.

As Equações (3a) e (3b) correspondem às restrições de balanço de potência ativa e reativa, respectivamente. $\mathrm{O}$ valor de posição $C H_{k m}\left(x_{k m}\right)$ é multiplicado pelas expressões dos fluxos de potência ativa $P_{k m}$ e reativa $Q_{k m}$.

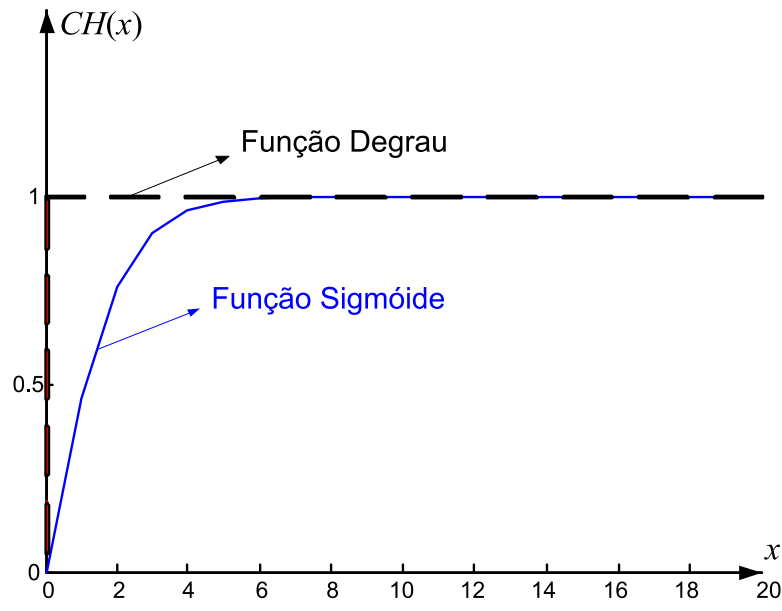

Figura 1: Função contínua de chaveamento.

Para a solução do problema de FPO formulado na Equação (2), utiliza-se o Método Primal Dual de Pontos Interiores (MPI) (Granville et al., 1996; Karmarkar and Ramakrishnan, 1991). Solucionando esse problema, os valores de posição das chaves manobráveis $\left(\mathrm{CH}_{k m}\right)$ encontram-se no intervalo contínuo $[0,1]$. Porém, na prática, os estados das chaves são discretos, ou seja, ligado $\left(\mathrm{CH}_{k m}=1\right)$ ou desligado $\left(\mathrm{CH}_{k m}=0\right)$. Logo, para obtenção de uma solução discreta para esse problema, deve-se adotar uma estratégia para definição dos estados das chaves. Uma das alternativas é calcular um índice de sensibilidade, de forma a determinar qual chave será manobrada.

A metodologia de restabelecimento ótimo proposta visa determinar qual chave manobrável deve ser fechada, de forma a restaurar energia para uma área do sistema inicialmente não alimentada devido a um defeito no sistema. A chave escolhida deve restabelecer a maior quantidade de carga possível. Baseado nesta premissa, o índice de sensibilidade proposto para determinação do estado das chaves corresponde a uma medida do impacto que o fechamento de determinado circuito causa na função objetivo do FPO. Este índice é dado por:

$$
I D X_{k m}=C H_{k m}\left(x_{k m}\right) \cdot S_{k m}
$$

Onde:

$$
\begin{array}{ll}
I D X_{k m} & \begin{array}{l}
\text { Representa o índice de sensibilidade para } \\
\text { definição do estado da chave } k-m ;
\end{array} \\
S_{k m} & \begin{array}{l}
\text { Fluxo de potência aparente entre as } \\
\text { barras } k-m .
\end{array}
\end{array}
$$

O índice proposto $\left(I D X_{k m}\right)$ na Equação (5) é proporcional ao valor de posição $C H_{k m}\left(x_{k m}\right)$. Quanto maior o valor de 
$\mathrm{CH}_{k m}\left(x_{k m}\right)$, maior é a tendência de fechamento do circuito $k-m$ para maximizar a carga não suprida, estabelecida pela função objetivo.

Adicionalmente, o índice $I D X_{k m}$ é diretamente proporcional ao fluxo de potência aparente entre as barras do circuito $k$ - $m$. Isto retrata a condição de que uma chave que transporta maior quantidade de potência aparente tem maior capacidade de restabelecer as cargas não supridas e deve ser priorizada para fechamento.

Considerando este aspecto, conclui-se que a chave preferencial para fechamento é aquela que possui o maior índice $I D X_{k m}$, pois seu fechamento resulta no restabelececimento da maior quantidade de carga possível. As chaves são operadas passo a passo, de forma a prover uma sequência viável e segura de operação do SDE.

O Algoritmo de Restabelecimento de Sistemas de Distribuição é apresentado pelo fluxograma da Figura 2.

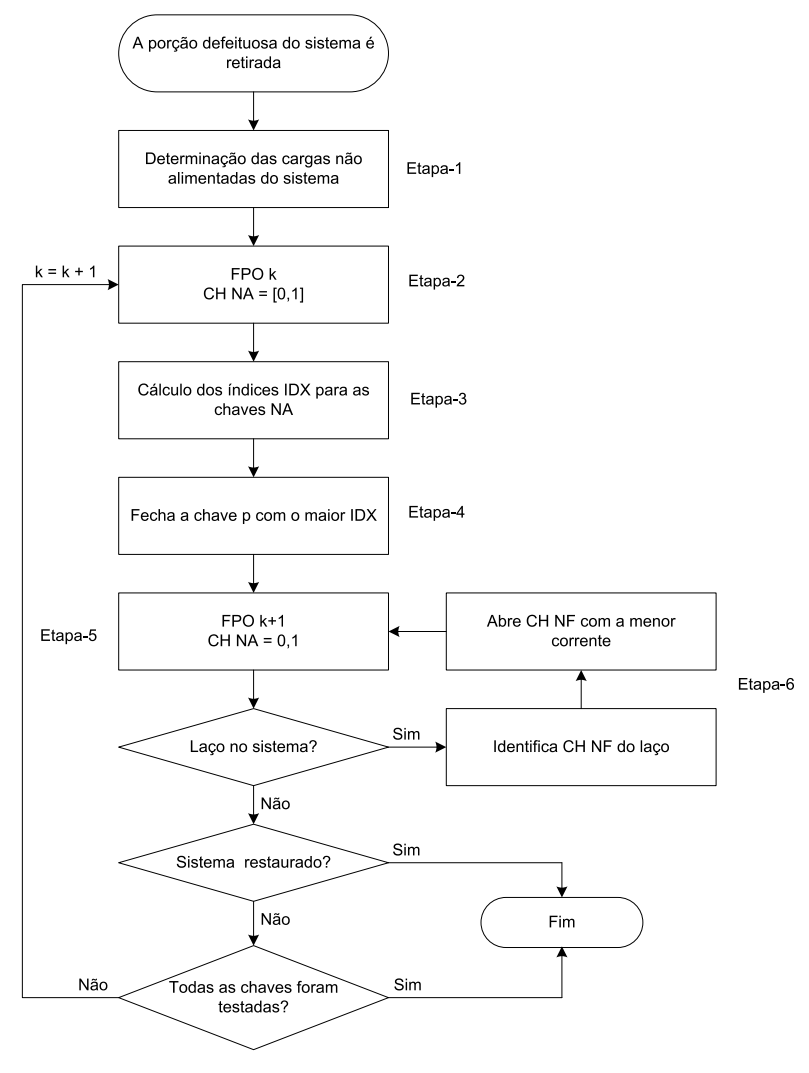

Figura 2: Fluxograma do algoritmo proposto.

O algoritmo proposto considera inicialmente a ocorrência de um defeito em um ou mais ramos do sistema. Nesta condição, o sistema de proteção atua e desliga toda a região a jusante do dispositivo de proteção. Em seguida, o ramo defeituoso é localizado e retirado do sistema. A partir desta configuração, as chaves NA são fechadas em sequência até que se consiga restabelecer toda a carga não suprida. As etapas deste algoritmo são descritas a seguir:

\section{Etapa-1}

O objetivo da Etapa-1 é determinar quais barras do sistema de distribuição não estão alimentadas após o defeito. Essas barras são determinadas utilizando-se processo de busca no grafo da rede. A cada carga localizada nessas barras é atribuída a variável variando de 0 a 1 , representando o corte de carga. O corte de carga multiplica os valores de carga ativa $\left(P l_{k}\right)$ e reativa $\left(Q l_{k}\right)$ dessas barras.

\section{Etapa-2}

A Etapa-2 do algoritmo tem como objetivo determinar os valores das variáveis necessários para o cálculo do índice de sensibilidade IDX, expresso pela Equação (5). Dessa forma, executa-se o FPO com a função objetivo dada pela Equação (2). Nesta etapa considera-se as variáveis associadas às chaves NA contínuas conforme Equação (4). Os valores de $x$ para estas chaves são iniciados com $x=0$ e após a simulação do FPO, eles encontram-se no intervalo contínuo $[0,1]$.

\section{Etapa-3}

Na Etapa-3 é efetuado o cálculo do índice de sensibilidade IDX para as chaves NA, conforme Equação (5). As variáveis necessárias para este cálculo são obtidas da solução do FPO da Etapa-2 no algoritmo. As chaves prioritárias para fechamento são listadas de acordo com a ordem decrescente dos índices IDX. A primeira chave dessa lista é a que possui o maior índice IDX e que, portanto, deve ser fechada.

\section{Etapa-4}

Na Etapa-4 a chave com o maior índice IDX é fechada. Nesta etapa, todas as chaves do sistema são transformadas em variáveis discretas, tendo seus valores fixados de acordo com o estado das mesmas: chave aberta $C H(x)=0$ e chave fechada $C H(x)=1$.

\section{Etapa-5}

O objetivo desta etapa é obter os valores de corte de carga $(\gamma)$, ou seja, diferente da Etapa-2, procura-se saber o estado da rede considerando o fechamento da chave definida na Etapa4. Para tanto, é executado um novo FPO considerando as chaves como variáveis discretas. Consequentemente os valores de $\gamma$ associados aos cortes de carga são obtidos, satisfazendo as restrições do problema. Em seguida, é verificado se o fechamento da chave cria um laço no sistema. Caso 
positivo, a Etapa-6 é executada de forma a eliminar o laço formado. Não havendo laços no sistema, os valores de $\gamma$ de cada barra inicialmente não alimentada são analisados. Caso todos os cortes de carga sejam iguais a 1 , as cargas não supridas do sistema foram restabelecidas e o algoritmo é encerrado. Caso contrário, é verificado se existem chaves NA que podem ser manobradas. Se existirem chaves NA manobráveis, o algoritmo retorna a Etapa-2. Se todas as chaves já tiverem sido manobradas, o algoritmo encerra indicando a quantidade de carga que foi restabelecida no sistema.

\section{Etapa-6}

Caso um laço seja identificado no sistema, todas as chaves NF pertencentes ao laço devem ser identificadas. Isso é feito utilizando o algoritmo de busca em grafos proposto por Balabanian and Bickart (1969). De acordo com Martin and Gil (2008), em um laço, a chave que causa o menor impacto nas perdas no sistema é aquela localizada nas proximidades da barra onde os fluxos de potência ativa $P$ e reativa $Q$ se invertem. A mesma analogia pode ser utilizada para o restabelecimento dos SDE. Através dos resultados obtidos na Etapa-5, é possível determinar a barra onde os fluxos de potência se invertem. A chave NF que deve ser aberta é aquela que possui o menor fluxo de potência passando através dela. Após a escolha da chave, a Etapa-5 é executada novamente.

\section{RESULTADOS}

Nesta seção são apresentados os resultados obtidos através da aplicação do algoritmo proposto de restabelecimento do SDE. Para tanto, são utilizados o sistemas de 16 barras proposto em (Civanlar et al., 1988) e o sistema de 33 barras apresentado por (Baran and Wu, 1989). Os resultados obtidos são comparados com os apresentados por Lin and Chin (1998). As simulações foram realizadas utilizando-se um microcomputador Intel Core I5, velocidade 2,66GHz, e o software Microsoft Visual ${ }^{\mathrm{R}} \mathrm{C}++$.

\subsection{Sistema teste de 16 Barras}

O sistema teste de 16 barras (Civanlar et al., 1988) é composto de 3 alimentadores de $23 \mathrm{kV}$ contendo 16 circuitos. As chaves NF dos circuitos são representadas por linhas contínuas. Esses circuitos estão acoplados por chaves normalmente abertas NA representadas por linhas tracejadas.

A Figura 3 apresenta a configuração de mínimas perdas proposta por Lin and Chin (1998).

Considerando a ocorrência de um defeito no ramo $\mathrm{S}_{5}$ (Figura 4), o método proposto irá apresentar uma solução passo a passo para o restabelecimento do sistema de distribuição

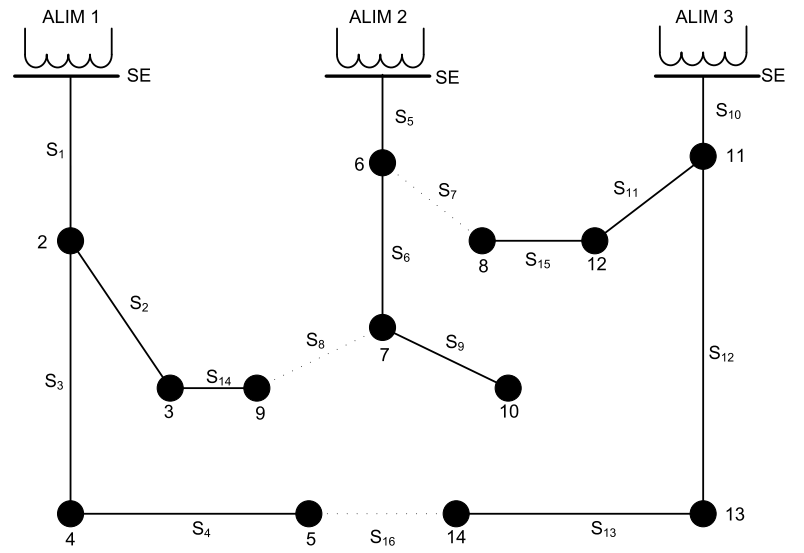

Figura 3: Sistema teste de 16 barras reconfigurado.

de energia. Neste primeiro estudo, a tensão da subestação é considerada igual a 1.0 p.u. e são adotados os limites de tensão de 0,95 p.u. e 1,05 p.u..

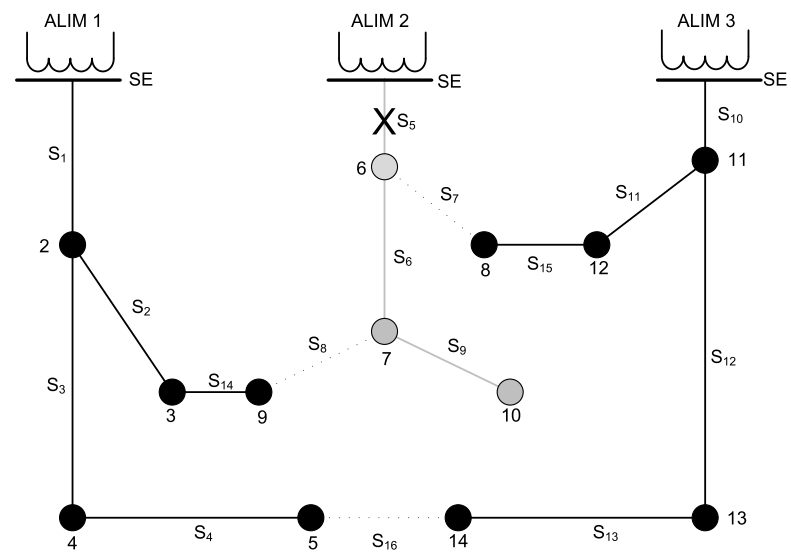

Figura 4: Defeito no sistema de 16 barras.

\section{Etapa-1}

O objetivo da Etapa-1 é a determinação das cargas não alimentadas do sistema. Após a retirada do ramo $\mathrm{S}_{5}$ verifica-se que as cargas conectadas as barras 6,7 e 10 ficam desalimentadas, deixando de suprir $13500 \mathrm{~kW}$ aos consumidores. A partir dessa informação é arbitrada uma variável de corte para cada barra, conforme Tabela 1.

\section{Etapa-2: $1^{\circ}$ execução:}

A Etapa-2 tem como objetivo otimizar o sistema através da simulação do FPO formulado em (2), maximizando a carga não suprida do sistema. Neste ponto, as chaves são tratadas com funções contínuas definidas em (4). 
Tabela 1: Corte de Carga do Sistema de 16 barras.

\begin{tabular}{|c|c|}
\hline Barra & Corte de Carga \\
\hline 6 & $\gamma_{1}$ \\
\hline 7 & $\gamma_{2}$ \\
\hline 10 & $\gamma_{3}$ \\
\hline
\end{tabular}

\section{Etapa-3: $1^{\circ}$ execução:}

Esta etapa efetua o cálculo dos índices de sensibilidade IDX, conforme Equação (5), para todas as chaves NA do sistema $\left(\mathrm{S}_{7}, \mathrm{~S}_{8}, \mathrm{~S}_{9}\right)$. Estes índices são apresentados na Tabela 2.

Tabela 2: Índices IDX sistema 16 barras - $1^{\text {a }}$ Execução.

\begin{tabular}{|c|c|}
\hline Chave & $I D X$ \\
\hline $\mathrm{S}_{7}$ & 0,012737 \\
\hline $\mathrm{S}_{8}$ & 0,091502 \\
\hline $\mathrm{S}_{16}$ & 0,004497 \\
\hline
\end{tabular}

\section{Etapa-4: $1^{\circ}$ execução:}

Na Etapa-4 somente a chave com o maior IDX da Tabela 2 é fechada. Dessa forma, pode-se avaliar o impacto da manobra no restabelecimento do sistema. Neste caso, a chave $\mathrm{S}_{8}$ é escolhida para ser fechada.

\section{Etapa-5: $1^{\circ}$ execução:}

Nesta etapa é simulado novamente o FPO formulado em (2) considerando a chave $S_{8}$ fechada e as chaves $S_{7}$ e $S_{16}$ abertas. Após a execução do FPO é verificado se o fechamento da chave $S_{8}$ causa a formação de um laço no sistema. Como pode ser visto na Figura 4, essa operação não fecha um laço no sistema.

Em seguida, é verificado se o fechamento da chave $\mathrm{S}_{8}$ restabelece o sistema. Conforme pode ser visto na Tabela 3, o sistema não foi totalmente recomposto. As cargas das barras 7 e 10 foram totalmente restauradas, enquanto somente $17,05 \%$ da carga da barra 6 foi suprida.

Em seguida, é verificado se existem outras chaves NA que podem restabelecer o sistema. As chaves $S_{7}$ e $S_{16}$ são chaves NA que podem ser manobradas. Dessa forma a Etapa-2 é executada novamente.
Tabela 3: Carga Restabelecida - $1^{\text {a }}$ Execução.

\begin{tabular}{|c|c|c|c|}
\hline Barra & $\begin{array}{c}\text { Carga } \\
(\mathrm{kW})\end{array}$ & $\begin{array}{c}\text { Corte de } \\
\text { Carga }\end{array}$ & $\begin{array}{c}\text { Carga } \\
\text { Restabelecida }(\mathrm{kW})\end{array}$ \\
\hline 6 & 4000 & $\gamma_{1}=0,1705$ & 682,28 \\
\hline 7 & 5000 & $\gamma_{2}=1$ & 5000 \\
\hline 10 & 4500 & $\gamma_{3}=1$ & 4500 \\
\hline
\end{tabular}

\section{Etapa-2: $2^{\circ}$ execução:}

A Etapa-2 é executada novamente através da simulação do (FPO) formulado em (2), maximizando a carga não suprida do sistema.

\section{Etapa-3: $2^{\circ}$ execução:}

São calculados os índices de sensibilidade IDX, conforme (5), para todas as chaves NA do sistema $\left(\mathrm{S}_{7}\right.$ e $\left.\mathrm{S}_{9}\right)$. Estes índices são apresentados na Tabela 4.

Tabela 4: Índice SS sistema 16 barras - $2^{\mathrm{a}}$ Execução.

\begin{tabular}{|c|c|}
\hline Chave & $I D X$ \\
\hline $\mathrm{S}_{7}$ & 0,013465 \\
\hline $\mathrm{S}_{16}$ & 0,000005 \\
\hline
\end{tabular}

\section{Etapa-4: $2^{\circ}$ execução:}

A chave $S_{7}$ é escolhida para ser fechada, pois possui o maior IDX da Tabela 4.

\section{Etapa-5: $2^{\circ}$ execução:}

O FPO formulado em (2) é simulado novamente, considerando as chaves $S_{7}$ e $S_{8}$ fechadas. Após a execução do FPO é verificado que o fechamento da chave $S_{7}$ causa a formação de um laço no sistema.

\section{Etapa-6: $2^{\circ}$ execução:}

Na Etapa-6 é identificado que as chaves $S_{1}, S_{2}, S_{6}, S_{7}$, $\mathrm{S}_{8}, \mathrm{~S}_{10}, \mathrm{~S}_{11}, \mathrm{~S}_{14}$, e $\mathrm{S}_{15}$ formam um laço no sistema. A partir dos resultados obtidos na Etapa-5 verifica-se que o sentido do fluxo de potência se inverte na barra 7 e que o menor fluxo é encontrado na chave $S_{6}$. Portanto, essa chave é escolhida para ser aberta. 


\section{Etapa-5: $3^{\circ}$ execução:}

O FPO formulado na Equação (2) é calculado, considerando as chaves $S_{7}$ e $S_{8}$ fechadas e a chave $S_{6}$ aberta.

Novamente é verificado se a nova configuração de chaves restabelece o sistema proposto.

Tabela 5: Carga Restabelecida - $3^{\text {a }}$ Execução.

\begin{tabular}{|c|c|c|c|}
\hline Barra & $\begin{array}{c}\text { Carga } \\
(\mathrm{kW})\end{array}$ & $\begin{array}{c}\text { Corte de } \\
\text { Carga }\end{array}$ & $\begin{array}{c}\text { Carga } \\
\text { Restabelecida }(\mathrm{kW})\end{array}$ \\
\hline 6 & 4000 & $\gamma_{1}=1$ & 4000 \\
\hline 7 & 5000 & $\gamma_{2}=1$ & 5000 \\
\hline 10 & 4500 & $\gamma_{3}=1$ & 4500 \\
\hline
\end{tabular}

Conforme Tabela 5, o sistema é 100\% restabelecido e a restrição de radialidade é obedecida. Durante todas as etapas do processo, os níveis de tensão foram mantidos dentro do limite estabelecido e as cargas na área não defeituosa foram atendidas.

Para comprovar a vantagem do método, a Tabela 6 mostra o comparativo do resultado obtido com o método proposto por Lin and Chin (1998).

Tabela 6: Comparação dos métodos para o sistema 16 barras.

\begin{tabular}{|c|c|c|}
\hline Método & Chaveamentos & $\begin{array}{c}\text { Carga } \\
\text { Restabelecida }\end{array}$ \\
\hline Lin and Chin (1998) & Fechar $S_{7}$ & $67 \%$ \\
\hline Proposto & $\begin{array}{c}\text { Fechar } S_{8} \\
\text { Fechar } S_{7} \\
\text { Abrir } S_{6}\end{array}$ & $100 \%$ \\
& \\
\hline
\end{tabular}

Na metodologia de Lin and Chin (1998), os autores consideram que a carga na barra 10 não é prioritária, podendo ser descartada. Dessa forma, o autor apresenta como resultado final o fechamento da chave $S_{7}$ restabelecendo somente a carga das barras 6 e 7. Na metodologia proposta, fechando-se inicialmente a chave $\mathrm{S}_{8}, 75,42 \%$ da carga não suprida é restaurada. Fechando-se em seguida a chave $\mathrm{S}_{7} 100 \%$ da carga é restabelecida. No entanto, um laço é formado no sistema. Abrindo-se a chave $S_{6}$ a restrição de radialidade é respeitada.

\subsection{Sistema teste de 33 Barras}

O sistema teste de 33 barras (Baran and Wu, 1989), 12,66 kV, carga total de $3715 \mathrm{~kW}$, é composto de 5 chaves de interconexão (NA). A Figura 5 representa a configuração proposta em Lin and Chin (1998), na qual as chaves NA são $S_{7}, S_{9}$, $S_{14}, S_{32}$ e $S_{37}$. A tensão na barra 33, que é a subestação, foi fixada em 1,0 p.u.

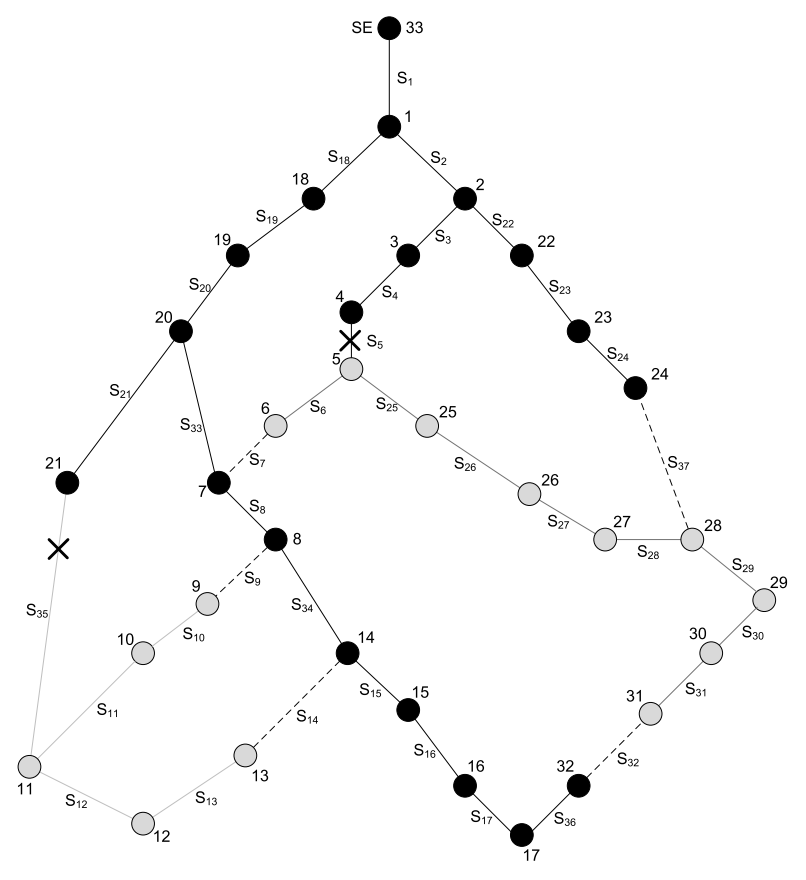

Figura 5: Sistema teste de 33 barras.

Nesse sistema são aplicados dois defeitos simultâneos nos ramos $S_{5}$ e $S_{35}$. Portanto, as cargas nas barras número 5, $6,9,10,11,12,13,25,26,27,28,29,30$ e 31 ficam sem alimentação e são representadas em cinza na Figura 5.

A Tabela 7 mostra a comparação entre o método apresentado por Lin and Chin (1998) e o algoritmo proposto nesse artigo usando diferentes limites de tensão nas barras.

Para o limite de tensão de 0,80 p.u. os dois métodos restabelecem o sistema completamente usando chaves diferentes. Para valores de 0,85 e 0,90 p.u., o algoritmo proposto restabelece $100 \%$ da carga enquanto o método de Lin and Chin (1998) restabelece 98\% e 79\% da carga respectivamente.

Considerando um limite de tensão mais restritivo de 0,93 p.u., a metodologia de Lin and Chin (1998) restabelece somente $43 \%$ da carga. Por outro lado, o algoritmo proposto restabelece $100 \%$ da carga através da operação de fechamento e abertura de chaves do sistema. Além disso, esta metodologia fornece uma sequência viável de operação para essas chaves $\left(1^{\circ}\right.$ : Fechar $S_{37}, 2^{\circ}$ : Fechar $S_{9}, 3^{\circ}$ : Fechar $S_{32}$, $4^{\circ}$ :Abrir $S_{36}, 5^{\circ}$ :Fechar $S_{7}$ e $6^{\circ}$ :Abrir $S_{6}$ ), garantindo uma condição de chaveamento segura para o sistema.

Devido à alteração do índice sensibilidade, os resultados desse trabalho são superiores aos apresentados pelos auto- 
Tabela 7: Solução do sistema 33 barras.

\begin{tabular}{|c|c|c|c|c|}
\hline \multirow{2}{*}{$\begin{array}{l}\text { Limite de } \\
\text { Tensão (pu) }\end{array}$} & \multicolumn{2}{|c|}{ Proposto } & \multicolumn{2}{|c|}{ Lin and Chin (1998) } \\
\hline & Chaveamento & $\begin{array}{c}\text { Carga } \\
\text { Restabelecida }\end{array}$ & Chaveamento & $\begin{array}{c}\text { Carga } \\
\text { Restabelecida } \\
\end{array}$ \\
\hline 0,80 & \multirow{3}{*}{$\begin{array}{l}\text { Fechar } S_{37} \\
\text { Fechar } S_{9}\end{array}$} & $100 \%$ & \multirow[b]{4}{*}{$\begin{array}{l}\text { Fechar } S_{7} \\
\text { Fechar } S_{9}\end{array}$} & $100 \%$ \\
\hline 0,85 & & $100 \%$ & & $98 \%$ \\
\hline 0,90 & & $100 \%$ & & $79 \%$ \\
\hline 0,93 & $\begin{array}{c}\text { Fechar } S_{37} \\
\text { Fechar } S_{9} \\
\text { Fechar } S_{32} \\
\text { Abrir } S_{36} \\
\text { Fechar } S_{7} \\
\text { Abrir } S_{6}\end{array}$ & $100 \%$ & & $43 \%$ \\
\hline
\end{tabular}

res em Borges et al. (2011), onde os índices de sensibilidade eram calculados utilizando-se os multiplicadores de lagrange associados às respectivas chaves. Os multiplicadores de lagrange foram utilizados com sucesso em Oliveira et al. (2009), contudo, para o problema de restabelecimento as heurísticas adotadas no trabalho supracitado não podem ser utilizadas no problema de restabelecimento, tornando a utilização dos multiplicadores de lagrange ineficiente.

\subsection{Desempenho computacional}

Conforme apresentado por Perez-Guerrero and Heydt (2008) o restabelecimento é um problema de operação em tempo real, sendo desejado que uma solução seja obtida, no máximo, na escala dos minutos.

A Tabela 8 apresenta os tempos computacionais médios para a solução do problema utilizando a metodologia proposta. Para os sistemas testados, o problema de reconfiguração da rede foi resolvido em tempos inferiores a 1 segundo. Tendo em vista que a ferramenta computacional ainda se encontra em fase de desenvolvimento pode-se concluir que o método proposto se mostra promissor para utilização na operação de sistemas elétricos de distribuição.

\section{CONCLUSÃO}

Este trabalho apresentou um algoritmo para o restabelecimento de sistemas de distribuição (SDE) utilizando fluxo de potência ótimo (FPO). O objetivo do problema é maximizar a carga das barras afetadas pelo defeito em um ou mais ramos do sistema. Considerando-se as elevadas dimensões dos SDE, trata-se de um problema de programação não linear inteira mista de difícil solução pelos métodos tradicionais de cálculo.
Tabela 8: Comparação dos métodos para o sistema 16 barras.

\begin{tabular}{|c|c|c|}
\hline Sistema & $\begin{array}{c}\text { Limite inferior de } \\
\text { tensão (pu) }\end{array}$ & Tempo (s) \\
\hline $\begin{array}{c}\text { 16 barras (Civanlar } \\
\text { et al., 1988) }\end{array}$ & 0,95 & 0,219 \\
\hline \multirow{3}{*}{$\begin{array}{c}\text { 33 barras (Baran } \\
\text { and Wu, 1989) }\end{array}$} & 0,80 & 0,552 \\
\cline { 2 - 3 } & 0,85 & 0,552 \\
\cline { 2 - 3 } & 0,90 & 0,552 \\
\hline
\end{tabular}

Ao contrário de outros métodos presentes na literatura, o algoritmo proposto fornece uma sequência de chaveamento viável para o sistema, permitindo uma operação segura a cada passo do restabelecimento. Outra característica que deve ser ressaltada é que o método considera a restrição de operação radial do sistema. Caso esta não seja uma restrição do problema em questão, a Etapa-6 do algoritmo pode ser suprimida. A metodologia trata de forma adequada os limites de tensão nas barras, pois utiliza FPO para solução do problema.

Os resultados obtidos para os sistemas simulados se mostraram superiores aos encontrados na literatura. O método proposto apresenta como vantagem adicional a solução do problema com menor esforço computacional com relação às metodologias desenvolvidas utilizando-se técnicas inteligentes (ex. Algoritmo Genético), devido ao reduzido número de execuções de FPO, favorecendo a aplicação do mesmo na operação de sistemas de grande porte. 


\section{AGRADECIMENTOS}

Os autores gostariam de agradecer o CNPq e FAPEMIG pelo apoio através do projeto INERGE (INCT de Energia Elétrica).

\section{REFERÊNCIAS}

Adibi, M., Clelland, P., Fink, L., Happ, H., Kafka, R., Raine, J., Scheurer, D. and Trefny, F. (1987). Power system restoration - a task force report, IEEE Transactions on Power Systems 2(2): 271-277.

Aoki, K., Nara, K., Itoh, M., Satoh, T. and Kuwabara, H. (1989). A new algorithm for service restoration in distribution systems, IEEE Transactions on Power Delivery 4(3): $1832-1839$.

Asakura, T., Yura, T., Hayashi, N. and Fukuyama, Y. (2000). Long-term distribution network expansion planning considering multiple construction plans, International Conference on Power System Technology, 2000. Proceedings. PowerCon 2000., Vol. 2, pp. 1101-1106.

Balabanian, N. and Bickart, T. A. (1969). Electrical Network Theory, Wiley, New York.

Baran, M. and Wu, F. (1989). Network reconfiguration in distribution systems for loss reduction and load balancing, IEEE Transactions on Power Delivery 4(2): 14011407.

Borges, T., Carneiro, S., Garcia, P., Pereira, J., de Oliveira, L. and da Silva, I. (2011). Distribution systems restoration using the interior point method and sensibility analysis, IEEE Power and Energy Society General Meeting, 2011, pp. 1-4.

Bretas, N., Delbem, A. and Carvallho, A. (2001). Representação por cadeias de grafo para ag aplicados ao restabelecimento ótimo em sistemas de distribuição radiais, SBA: Controle \& Automação 12(1): 42-51.

Bryson, W. C. and Hayward, A. P. (1940). Restoration of service on a metropolitan power system, Transactions of the American Institute of Electrical Engineers 59(10): 563-570.

Celli, G., Pilo, F., Pisano, G., Allegranza, V., Cicoria, R. and Iaria, A. (2004). Meshed vs. radial mv distribution network in presence of large amount of dg, IEEE PES Power Systems Conference and Exposition, 2004., Vol. 2, pp. 709-714.

Chavali, S., Pahwa, A. and Das, S. (2002). A genetic algorithm approach for optimal distribution feeder restoration during cold load pickup, Proceedings of the
2002 Congress on Evolutionary Computation, 2002. CEC '02., Vol. 2, pp. 1816-1819.

Chen, C.-S., Lin, C.-H. and Tsai, H.-Y. (2002). A rule-based expert system with colored petri net models for distribution system service restoration, IEEE Transactions on Power Systems 17(4): 1073-1080.

Chen, W.-H. (2010). Quantitative decision-making model for distribution system restoration, IEEE Transactions on Power Systems 25(1): 313-321.

Civanlar, S., Grainger, J., Yin, H. and Lee, S. (1988). Distribution feeder reconfiguration for loss reduction, IEEE Transactions on Power Delivery 3(3): 1217-1223.

da Silva, I., Carneiro, S., de Oliveira, E., de Souza Costa, J., Rezende Pereira, J. and Garcia, P. (2008). A heuristic constructive algorithm for capacitor placement on distribution systems, IEEE Transactions on Power Systems 23(4): 1619-1626.

Delbem, A., Bretas, N. and Carvallho, A. (2000). Algoritmo de busca com heurísticas fuzzy para restabelecimento de energia em sistemas radiais de distribuição, $S B A$ : Controle e Automação 11(1): 55-60.

Duque, O. and Morinigo, D. (2006). Load restoration in electric distribution networks using a metaheuristic technique, IEEE Mediterranean Electrotechnical Conference, 2006. MELECON 2006., pp. 1040-1043.

Fukuyama, Y. and Chiang, H.-D. (1995). A parallel genetic algorithm for service restoration in electric power distribution systems, Proceedings of 1995 IEEE International Conference on International Joint Conference of the Fourth IEEE International Conference on Fuzzy Systems and The Second International Fuzzy Engineering Symposium., Vol. 1, pp. 275-282.

Gomes, F. V., Pereira, J., Garcia, P., Vinagre, M. and Carneiro Jr, S. (2006). Reconfiguração de sistemas de distribuição utilizando fluxo de potência ótimo e análise de sensibilidade, SBA: Controle \& Automação 17(4): 469477.

Gomes, P. (2008). Segurança elétrica do sin, Tutorial sobre recomposição de sistemas, CEPEL, Rio de Janeiro. 1 CD-ROM.

Granville, S., Mello, J. and Melo, A. (1996). Application of interior point methods to power flow unsolvability, IEEE Transactions on Power Systems 11(2): 10961103.

Hsu, Y.-Y. and Huang, H.-M. (1995). Distribution system service restoration using the artificial neural network 
approach and pattern recognition method, IEE Proceedings on Generation, Transmission and Distribution 142(3): 251-256.

Hsu, Y.-Y., Huang, H.-M., Kuo, H.-C., Peng, S., Chang, C., Chang, K., Yu, H., Chow, C. and Kuo, R. (1992). Distribution system service restoration using a heuristic search approach, IEEE Transactions on Power Delivery 7(2): 734-740.

Hsu, Y.-Y. and Kuo, H.-C. (1994). A heuristic based fuzzy reasoning approach for distribution system service restoration, IEEE Transactions on Power Delivery 9(2): 948-953.

Huang, C.-M. (2003). Multiobjective service restoration of distribution systems using fuzzy cause-effect networks, IEEE Transactions on Power Systems 18(2): 867-874.

Kaewmanee, J. and Sirisumrannukul, S. (2011). Multiobjective service restoration in distribution system using fuzzy decision algorithm and node-depth encoding, 8th International Conference on Electrical Engineering/Electronics, Computer, Telecommunications and Information Technology (ECTI-CON), 2011, pp. 893896.

Karmarkar, N. K. and Ramakrishnan, K. G. (1991). Computational results of an interior point algorithm for large scale linear programming, Mathematical Programming 52(1-3): 555-586.

Kumar, V., Gupta, R. and Gupta, H. (2006). Stepwise restoration of power distribution network under cold load pickup, International Conference on Power Electronics, Drives and Energy Systems, 2006. PEDES '06., pp. 15.

Kumar, Y., Das, B. and Sharma, J. (2006). Genetic algorithm for supply restoration in distribution system with priority customers, International Conference on Probabilistic Methods Applied to Power Systems, 2006. PMAPS 2006., pp. 1-7.

Kuo, H.-C. and Hsu, Y.-Y. (1993). Distribution system load estimation and service restoration using a fuzzy set approach, IEEE Transactions on Power Delivery 8(4): 1950-1957.

Lei, J., Deng, Y., He, Y. and Zhang, B. (2000). Network reconfiguration in unbalanced distribution systems for service restoration and loss reduction, IEEE Power Engineering Society Winter Meeting, 2000., Vol. 4, pp. 2345-2350.
Li, X., Xu, Y. and Zhang, L. (2009). Distribution service restoration with dgs based on multi-agent immune algorithm, 2nd International Conference on Power Electronics and Intelligent Transportation System (PEITS), 2009, Vol. 1, pp. 1-4.

Lim, S.-I., Lee, S.-J., Choi, M.-S., Lim, D.-J. and Ha, B.-N. (2006). Service restoration methodology for multiple fault case in distribution systems, IEEE Transactions on Power Systems 21(4): 1638-1644.

Lin, W.-M. and Chin, H.-C. (1998). A new approach for distribution feeder reconfiguration for loss reduction and service restoration, IEEE Transactions on Power Delivery 13(3): 870-875.

Liu, C.-C., Lee, S. and Venkata, S. (1988). An expert system operational aid for restoration and loss reduction of distribution systems, IEEE Transactions on Power Systems 3(2): 619-626.

Luan, W., Irving, M. and Daniel, J. (2002). Genetic algorithm for supply restoration and optimal load shedding in power system distribution networks, IEE Proceedings on Generation, Transmission and Distribution 149(2): 145-151.

Martin, J. and Gil, J. (2008). A new heuristic approach for distribution systems loss reduction, Electric Power Systems Research 78(11): 1953-1958.

Mathias-Neto, W., Leão, F. and Mantovani, J. (2010). Distribution system restoration in a dg environment using a heuristic constructive multi-start algorithm, IEEE/PES Transmission and Distribution Conference and Exposition: Latin America (T D-LA), 2010, pp. 86-91.

Miu, K., Chiang, H.-D., Yuan, B. and Darling, G. (1998). Fast service restoration for large-scale distribution systems with priority customers and constraints, IEEE Transactions on Power Systems 13(3): 789-795.

Momoh, J. and Caven, A. (2003). Distribution system reconfiguration scheme using integer interior point programming technique, IEEE PES Transmission and Distribution Conference and Exposition, 2003, Vol. 1, pp. 234241.

Morelato, A. and Monticelli, A. (1989). Heuristic search approach to distribution system restoration, IEEE Transactions on Power Delivery 4(4): 2235-2241.

Mun, K. J., Park, J., Kim, H.-S. and Seo, J.-I. (2001). Development of real-time-service restoration system for distribution automation system, IEEE International Symposium on Industrial Electronics, 2001. Proceedings. ISIE 2001., Vol. 3, pp. 1514-1519. 
Oliveira, L., Jr., S. C., de Oliveira, E. J., Pereira, J., Jr., I. C. S. and Costa, J. S. (2010). Optimal reconfiguration and capacitor allocation in radial distribution systems for energy losses minimization, International Journal of Electrical Power \& Energy Systems 32(8): 840-848.

Oliveira, L. W., C. Junior, S., Costa, J. S., Oliveira, E. J. d., Pereira, J. A. L. R. and S. Junior, I. C. (2009). Reconfiguração ótima de sistemas de distribuição para minimização de perdas de energia, SBA: Controle \& Automação 20(2): 233-246.

Peponis, G. and Papadopoulos, M. (1995). Reconfiguration of radial distribution networks: application of heuristic methods on large-scale networks, IEE Proceedings on Generation, Transmission and Distribution 142(6): 631-638.

Perez-Guerrero, R. and Heydt, G. (2008). Distribution system restoration via subgradient-based lagrangian relaxation, IEEE Transactions on Power Systems 23(3): 1162-1169.

Popovic, D. and Popovic, Z. (2004). A risk management procedure for supply restoration in distribution networks, IEEE Transactions on Power Systems 19(1): 221-228.

Rodriguez, J. and Vargas, A. (2005). Fuzzy-heuristic methodology to estimate the load restoration time in mv networks, IEEE Transactions on Power Systems 20(2): 1095-1102.

Sarma, N., Prasad, V., Prakasa Rao, K. and Sankar, V. (1994). A new network reconfiguration technique for service restoration in distribution networks, IEEE Transactions on Power Delivery 9(4): 1936-1942.

Shirmohammadi, D. (1992). Service restoration in distribution networks via network reconfiguration, IEEE Transactions on Power Delivery 7(2): 952-958.

Tian, Y., Xin, J., Du, Z., Lin, T. and Cao, J. (2010). On the strategy of distribution system service restoration considering distributed generation, China International Conference on Electricity Distribution (CICED), 2010, pp. 1-5.

Trovato, M., Delvechio, G. and Bualoti, R. (1999). A fuzzy reasoning approach for distribution automation, International Conference on Electric Power Engineering, 1999. PowerTech Budapest 99., p. 249.

Tsai, M.-S. and Wu, W.-C. (2002). Development of an object-oriented expert system for multi-period load transfer, IEEE/PES Transmission and Distribution Conference and Exhibition 2002: Asia Pacific., Vol. 2, pp. 1170-1174.

Xianchao, H., Lizi, Z. and Taylor, G. (2010). Service restoration of distribution system with distributed generation, International Conference on Power System Technology (POWERCON), 2010, pp. 1-5.

Yi-Xiong, Y., Yan, W. and Yong-Sheng, S. (2011). The research on fault restoration considering distributed generation based particle swarm optimization, Asia-Pacific Power and Energy Engineering Conference (APPEEC), 2011, pp. 1-4. 